\title{
An experimental sensitivity comparison of the schlieren and background-oriented schlieren techniques applied to hypersonic flow \\ DOI:
}

10.1088/1361-6501/ab1582

\section{Document Version}

Accepted author manuscript

Link to publication record in Manchester Research Explorer

Citation for published version (APA):

Fisher, T., Quinn, M. K., \& Smith, K. (2019). An experimental sensitivity comparison of the schlieren and background-oriented schlieren techniques applied to hypersonic flow. Measurement Science and Technology. https://doi.org/10.1088/1361-6501/ab1582

\section{Published in:}

Measurement Science and Technology

\section{Citing this paper}

Please note that where the full-text provided on Manchester Research Explorer is the Author Accepted Manuscript or Proof version this may differ from the final Published version. If citing, it is advised that you check and use the publisher's definitive version.

\section{General rights}

Copyright and moral rights for the publications made accessible in the Research Explorer are retained by the authors and/or other copyright owners and it is a condition of accessing publications that users recognise and abide by the legal requirements associated with these rights.

\section{Takedown policy}

If you believe that this document breaches copyright please refer to the University of Manchester's Takedown Procedures [http://man.ac.uk/04Y6Bo] or contact uml.scholarlycommunications@manchester.ac.uk providing relevant details, so we can investigate your claim.

\section{OPEN ACCESS}


ACCEPTED MANUSCRIPT

\section{An experimental sensitivity comparison of the schlieren and background- oriented schlieren techniques applied to hypersonic flow}

To cite this article before publication: Tom Fisher et al 2019 Meas. Sci. Technol. in press https://doi.org/10.1088/1361-6501/ab1582

\section{Manuscript version: Accepted Manuscript}

Accepted Manuscript is "the version of the article accepted for publication including all changes made as a result of the peer review process, and which may also include the addition to the article by IOP Publishing of a header, an article ID, a cover sheet and/or an 'Accepted Manuscript' watermark, but excluding any other editing, typesetting or other changes made by IOP Publishing and/or its licensors"

This Accepted Manuscript is @ 2019 IOP Publishing Ltd.

During the embargo period (the 12 month period from the publication of the Version of Record of this article), the Accepted Manuscript is fully protected by copyright and cannot be reused or reposted elsewhere.

As the Version of Record of this article is going to be / has been published on a subscription basis, this Accepted Manuscript is available for reuse under a CC BY-NC-ND 3.0 licence after the 12 month embargo period.

After the embargo period, everyone is permitted to use copy and redistribute this article for non-commercial purposes only, provided that they adhere to all the terms of the licence https://creativecommons.org/licences/by-nc-nd/3.0

Although reasonable endeavours have been taken to obtain all necessary permissions from third parties to include their copyrighted content within this article, their full citation and copyright line may not be present in this Accepted Manuscript version. Before using any content from this article, please refer to the Version of Record on IOPscience once published for full citation and copyright details, as permissions will likely be required. All third party content is fully copyright protected, unless specifically stated otherwise in the figure caption in the Version of Record.

View the article online for updates and enhancements. 


\title{
An experimental sensitivity comparison of the schlieren and background-oriented schlieren techniques applied to hypersonic flow
}

\author{
Fisher, T.B., Quinn, M.K., Smith, K. L. 2018
}

School of Mechanical, Aerospace and Civil Engineering, The University of Manchester, United Kingdom. Corresponding author tom.fisher@manchester.ac.uk

\section{Abstract}

An experimental study was performed to evaluate the spatial resolution, sensitivity and dynamic range of schlieren and background-oriented schlieren (BOS) applied to hypersonic flows with multiple density gradients. For a single Mach number, shock profile extracts for three values of freestream density are compared. Three processing metrics are proposed for BOS to optimise the sharpness of captured shocks and minimise processing blur are presented. The BOS results have a lower spatial resolution and sharpness than the schlieren images, but captured the model flow field features that were resolved by schlieren. A higher dynamic range was afforded for BOS due to the user-determined synthetic knife edge cut-off.

\section{Introduction}

The background-oriented schlieren (BOS), method developed by Meier [1, 2] and Sutherland et al. $[3,4]$ exploits the same density gradient induced refractive index change used in traditional optical schlieren to produce a "synthetic schlieren" image. The term "backgroundoriented" refers to the apparent displacements in a background, upon which the camera focus is set, imaged through a region containing optical inhomogeneities. The technique uses an illuminated pattern [5], grid [6] or gradient [7], as a background which is imaged using a camera. Two images are taken; an undisplaced "wind off" reference image and a second, or series of "wind on" when a density gradient exists in the region between the camera and the background. Displacements between the two images of the background pattern resulting from shockwaves, expansions, and other density gradients are tracked using cross-correlation or optical flow methods. The two displacement fields in the vertical 
and horizontal directions can be related to the density field through the Gladstone-Dale relationship, [8] in equation 1 . The refractive index, $n$, is thereby related to the density of the air, $\boldsymbol{\rho}$, and the Gladstone-Dale constant, $\boldsymbol{k}$. The displacement vector fields can then be displayed as an image of the flow, without the need for expensive and precise parabolic mirrors and other optical elements [8]

$$
\boldsymbol{n}=\boldsymbol{k} \cdot \boldsymbol{\rho}+\mathbf{1}(1)
$$

Image displacement tracking has become routine through particle image velocimetry (PIV) with its widespread application to study aerodynamic phenomena [9]. PIV cross-correlation algorithms are often applied to process BOS images [10, 9] with studies performed by Atcheson et al. using optical flow-based algorithms for BOS [11]. The review paper by Raffel [12] details many different applications of BOS describing its origins and suitability of application to a variety of flows. BOS has been compared to schlieren to study transient shock interactions in free-flight within a wind tunnel [13]. Heineck et al. demonstrated the scalability of BOS to study to supersonic aircraft in flight. This implementation termed AirBOS, used the desert floor at Edwards Air Force base as the background and placed the camera optics in a Beechcraft B-200 Super King Air [14].

Experimental sensitivity studies have used simple flow cases to evaluate the technique at a variety of compressible Mach numbers. Transonic wind tunnel studies with values of freestream static density $\rho_{\infty} \cong 1.255 \mathrm{~kg} / \mathrm{m}^{3}$ on a cone-cylinder model were performed by Manovski et al. [15] for Mach numbers between 0.6 to 1.2. Extracted refraction angles for both BOS and schlieren found the minimum detectability for both techniques were \pm 1 arcsecond. Hypersonic flows in experimental facilities have much lower freestream static density, of the order $1 \times 10^{-1} \leq \rho_{\infty} \leq 1 \times 10^{-3} \mathrm{~kg} / \mathrm{m}^{3}$ or even lower, for very rarefied, continuum flows. Hypersonic BOS testing by Kirmse et al. [16] compared ray-traced CFD results of a sphere with BOS results around a sphere in Mach 6 flow. Good agreement was found for $80 \%$ of the extracted horizontal displacement profile for value of freestream static density of $\rho_{\infty} \cong 1.7 \times 10^{-3} \mathrm{~kg} / \mathrm{m}^{3}$. A further study by Rammanah et al. [17] studied a sharp 
$20^{\circ}$ circular cone and a re-entry body at Mach 4 in the Drummond reflected shock tunnel, and processed the results using a PIV algorithm. The static density value for these tests was $\rho_{\infty} \cong 0.13 \mathrm{~kg} / \mathrm{m}^{3}$. Shock angles measured from the processed BOS images were within $0.5^{\circ}$ of theory and matched well with CFD results.

Studies extant in the literature have explored either a single test condition or examined the influence of the freestream static density on the ability of BOS to capture different shock profiles. Experimental studies have not yet examined the effect of several values of freestream static density for a complex flow-field for a fixed Mach number. Furthermore, studies have not explored metrics to determine the optimum processing algorithm which maximises shock capturing but minimises processing blur. This study seeks to address these issues.

\section{Experimental tools and procedures}

An experimental campaign was designed using a model to generate a variety of common hypersonic flow patterns of varying strength. In optical schlieren the sensitivity and dynamic range are determined by the knife edge cut-off; with strong features typically clipped at the high-range and weaker features omitted. The knife edge orientation and cut-off are set prior to testing and cannot be adjust in post-processing. A compromise cut-off of $50 \%$ was used to capture all density gradients in a single image. In the BOS testing results from one density case are subjected to different processing window sizes. This enables the impact of the processing algorithms on the spatial resolution to be found.

A commercial Nikon D5200 24 megapixel DSLR camera is used for both techniques.

\subsection{Hypersonic wind tunnel and model}

The studies were performed using the University of Manchester's High Super-Sonic wind Tunnel (HSST). The facility is a long-duration blow-down tunnel capable of Mach numbers of $M=4.0,5.0,6.0$. For Mach 5.0 a maximum total pressure $\left(P_{0}\right)$ of $850 \mathrm{kPa}$ and total temperature $\left(T_{0}\right)$ range from $300 \mathrm{~K}$ through to $950 \mathrm{~K}$ are achievable with stable test times up 
to 7.5 seconds. A schematic of the facility is provided in Figure 1 with the major operating components labelled.

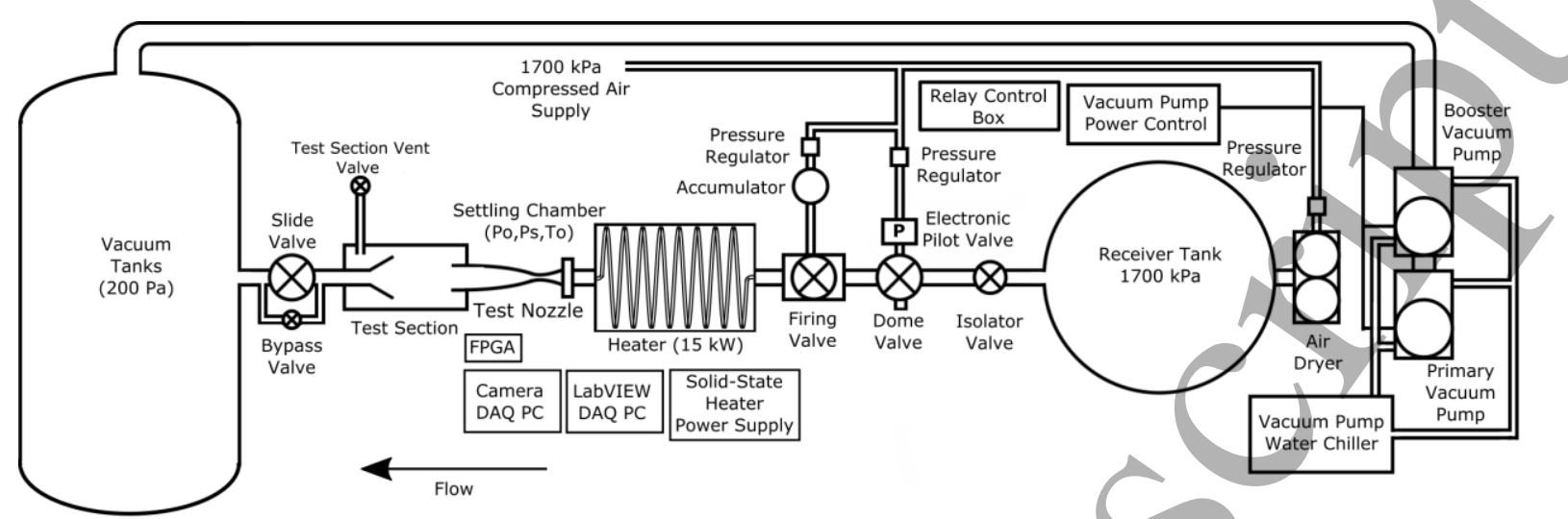

Figure 1: HSST facility schematic

Optical access to the plenum chamber around the $152 \mathrm{~mm}$ exit diameter free-jet nozzle is afforded through two parallel rectangular quartz windows ( 300 by $210 \mathrm{~mm}$ ) which span the full length of the useful test jet. A floor-mounted rail and an arc balance sting provide fixed model mounting locations.

A flared-cone model (Figure 2) was selected to generate an attached conical shock, flow expansions, a strong shock interaction, and a shear layer. The model was mounted at zero incidence angle of attack using an inclinometer.

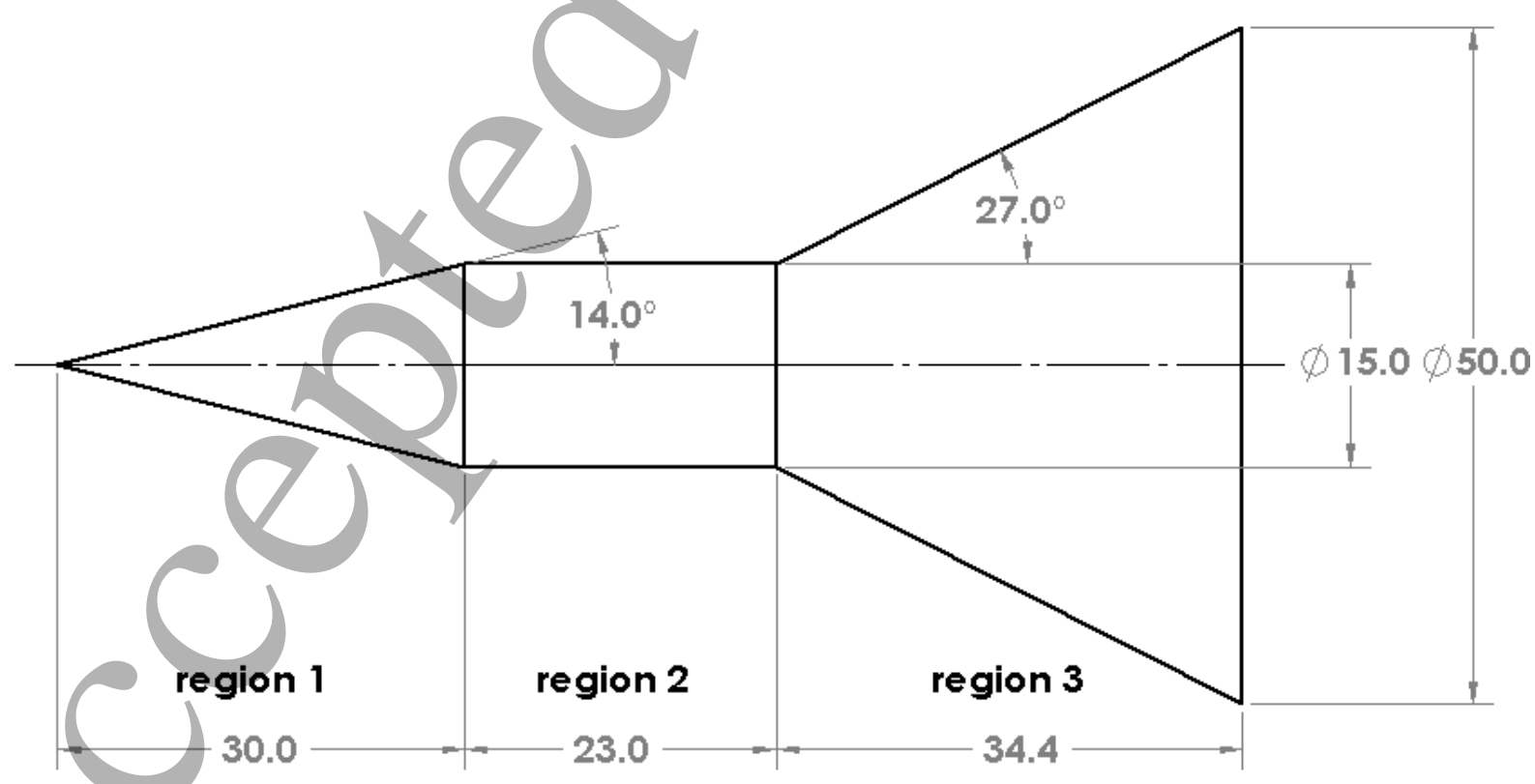

Figure 2: Flared-cone model - all dimensions in $\mathrm{mm}$ 
A Mach number of 5.0 was chosen for this test campaign as this provided an order of magnitude change of density over the operational range of the heater for a constant total pressure. Three flow conditions were used for the tests; termed the high-, medium-, and lowdensity cases. The flow conditions are given in Table 1 with Sutherlands' viscosity model used for Reynolds number calculation [18]. Variation of the conditions between test runs was found to be a maximum of $\pm 0.5 \%$.

Table 1: Test case flow conditions

\begin{tabular}{|c|c|c|c|}
\hline Test conditions/ test case & High-density & Medium-density & Low-density \\
\hline Total pressure $\left(\mathrm{P}_{\mathrm{o}}\right)(\mathrm{kPa})$ & 813.00 & 847.00 & 852.00 \\
\hline Total temperature $\left(\mathrm{T}_{\mathrm{o}}\right)(\mathrm{K})$ & 301.00 & 598.00 & 852.00 \\
\hline Static density $\left(\mathrm{kg} / \mathrm{m}^{3}\right)$ & 0.11 & 0.06 & 0.04 \\
\hline Unit Reynolds No $(\mathrm{Re} \mathrm{M} / \mathrm{m})$ & 23.92 & 8,16 & 4.57 \\
\hline
\end{tabular}

\subsection{BOS technique}

The BOS optical path was arranged around the HSST shown in Figure 3, with flow direction from top to bottom. Introducing a density gradient in the test section causes the light ray carrying the information of point $A$ on the background pattern to be refracted by a small angle, $\varepsilon x$, which strikes the sensor plane at position $A^{\prime}$ with an apparent displacement, $\Delta x$, between the "wind off" and "wind on" image pair. The magnitude of this apparent displacement is determined by the distances between the background-camera lens, $Z_{B}$, and the density gradient-lens, $Z_{A}$, typically expressed as a ratio $Z_{A} / Z_{B}$. Values of $Z_{A} / Z_{B} \cong 0.5$ generate large displacements but place the test model in a region of high blur. To preserve optical sharpness of the model and shockwaves BOS images require large depth of field to place the foreground and background in a similar focal plane. This is achieved with large $f_{\#}$, typically $f 22$, and $Z_{A} / Z_{B}$ ratio values between $0.7-0.8$ to compromise between maximising displacement and minimising optical blur [24, 25].

Sensitivity (S) and magnification (M) of the BOS system is determined by the focal length $(F)$ of the lens and magnification of the background as defined by Gojani et al. [19] were used 
to calculate the system parameters in Table 2. For this arrangement the background pattern magnification obtains 50 pixels per $\mathrm{mm}$ on the camera sensor plane.

Table 2: BOS parameters

\begin{tabular}{|l|l|}
\hline$Z_{D}$ & $260 \mathrm{~mm}$ \\
\hline$Z_{A}$ & $607 \mathrm{~mm}$ \\
\hline$Z_{B}$ & $867 \mathrm{~mm}$ \\
\hline$Z_{A} / Z_{B}$ & 0.70 \\
\hline$Z_{i}$ & $227 \mathrm{~mm}$ \\
\hline$F$ & $210 \mathrm{~mm}$ \\
\hline$f_{\#}$ & 22 \\
\hline$M$ & 0.26 \\
\hline$S$ & 68 \\
\hline
\end{tabular}

A single wavelet-type background pattern, described by Atcheson et al. [11] provides a multiscaled pattern with high frequency content at all scales. A background was generated comprising a $35 \%$ weighted random distribution of red, green and blue pixels. This was colour laser printed at 600 dpi with a scale of 20 pixels per mm on to A3 paper and held between two transparent acrylic plates.

The background was rear-illuminated by a custom LED array, comprising $600 \mathrm{JKL}$ Components ZFS-155000 white LEDs running at $24 \mathrm{~V} \mathrm{DC}$. The light source is positioned behind the test section window, shown in Figure 3, attached to a vibration-isolating aluminium profile frame. This isolates the optical path from the physical structure of the tunnel. The Nikon camera with a Nikon AF-S 28-300mm f/3.5-5.6 G ED VR lens was mounted on the frame positioned on the opposite side of the test section in Figure 3; the frame allows the camera to translate in three directions to set the desired field of view (FOV) of $118 \times 82 \mathrm{~mm}$. Background focus and triggering used the Nikon camera control software to obtain two wind-off and six wind-on images per run and recorded in raw file format. 


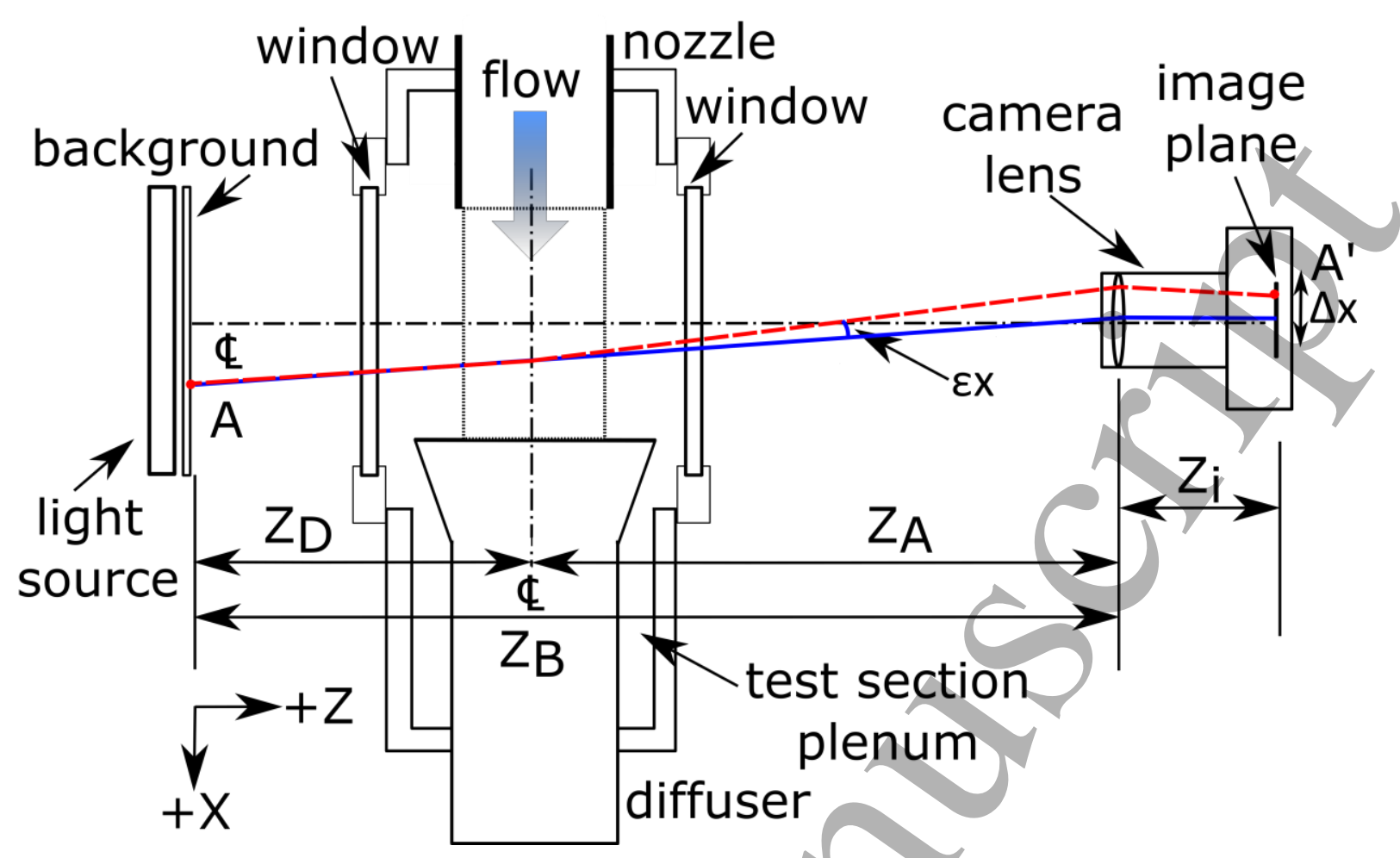

Figure 3: Schematic of the BOS technique implementation in the HSST

The camera was operated in aperture priority mode, with an exposure of $1 / 8$ seconds. This was due to the required large $\mathrm{f}_{\#}$ and illumination intensity restrictions of the lamp assembly.

The LaVision DaVis StrainMaster software, version 8.4.0, in 2D DIC LSM time series strain solver mode was used to process the BOS image data with displacements calculated relative to a single wind-off image. The raw image files are converted to 8 bit .tiff images using DC RAW and are read into DaVis. The region of interest (ROI) in the flow field was defined using a geometric mask. Algorithmic masking is used to define the area occupied by the test model and exclude this region from processing. A digital image correlation (DIC) least squares method (LSM) algorithm is used within the software to track the flow-induced displacements. The vector field outputs were exported in proprietary DaVis .vc7 format and imported into Matlab to generate the flow images. The horizontal displacement vector fields $\Delta \mathrm{X}$ are equivalent to the vertical cut-off knife edge employed for the conventional optical schlieren. Vertical vector displacements $\Delta \mathrm{Y}$ are equivalent to a horizontal knife edge, and 
the displacement vector magnitude $\Delta \mathrm{M}$ is equivalent to a pinhole schlieren image. The user is free to set the display limits to control the strength of shock features in a BOS image. Increasing the display range is equivalent to decreasing the cut-off of the synthetic BOS knife edge and hence a weaker equivalent optical schlieren knife edge cut-off and viceversa.

\subsection{0ptical schlieren}

Schlieren images were acquired through Töepler's Z-type schlieren method with a $50 \%$ vertical knife cut off. The same Nikon DSLR camera with a 1/4000 second exposure was used for image acquisition in raw file format. Two 12 inch diameter $f / 7.9$ mirrors pass the light from a $450 \mathrm{~W}$ Xenon arc lamp onto the knife edge in the cut-off plane which is then focused though a $500 \mathrm{~mm}$ achromatic doublet lens onto the camera sensor. The FOV was held constant at $120 \times 76 \mathrm{~mm}$.

\section{Results and discussion}

The results of the optical schlieren tests are presented in section 3.1, including a description of the flow field. The processing optimisation of BOS results is presented from section 3.2 with the synthetic knife edge orientations are shown in section 3.3. Non-dimensionalised line extracts of BOS pixel displacement and schlieren image intensity are then compared in section 3.4 to assess the dynamic range of BOS in comparison to schlieren.

\subsection{Optical schlieren results}

The flow field around the model is shown in Figure 4; the vertical orientation of the knife edge results in flow compressions as dark regions and expansions as light regions. Measurements of the cone angle using the ImageJ software indicate a $19.8^{\circ}$ shock angle from a $14^{\circ}$ cone half-angle. This value agrees within $0.7^{\circ}$ degrees with conical flow theory [20]. Two expansion regions can be seen on the model, a small region where the leading cone meets the cylindrical region 2 of the model, and on the edge of the larger flare at the base of the model in region 3 . The angle of the flare causes an adverse pressure gradient resulting in separation of the upstream laminar boundary layer after the first expansion fan. A 
free-shear layer bounds the recirculating flow at the model surface and the flow within the conical shock exiting the expansion region.

The impingement of the shear layer on the rear flared-cone surface produces an unsteady shock reflection, and strong recompression in the flow [21]. The reflected compression shock extends beyond the leading conical shock increasing the conical shock angle in the region behind the recompression. The strong recompression region is immediately followed by a strong expansion region centred on the vertex of the model flare, the flow expanding around into the base flow region behind the model.

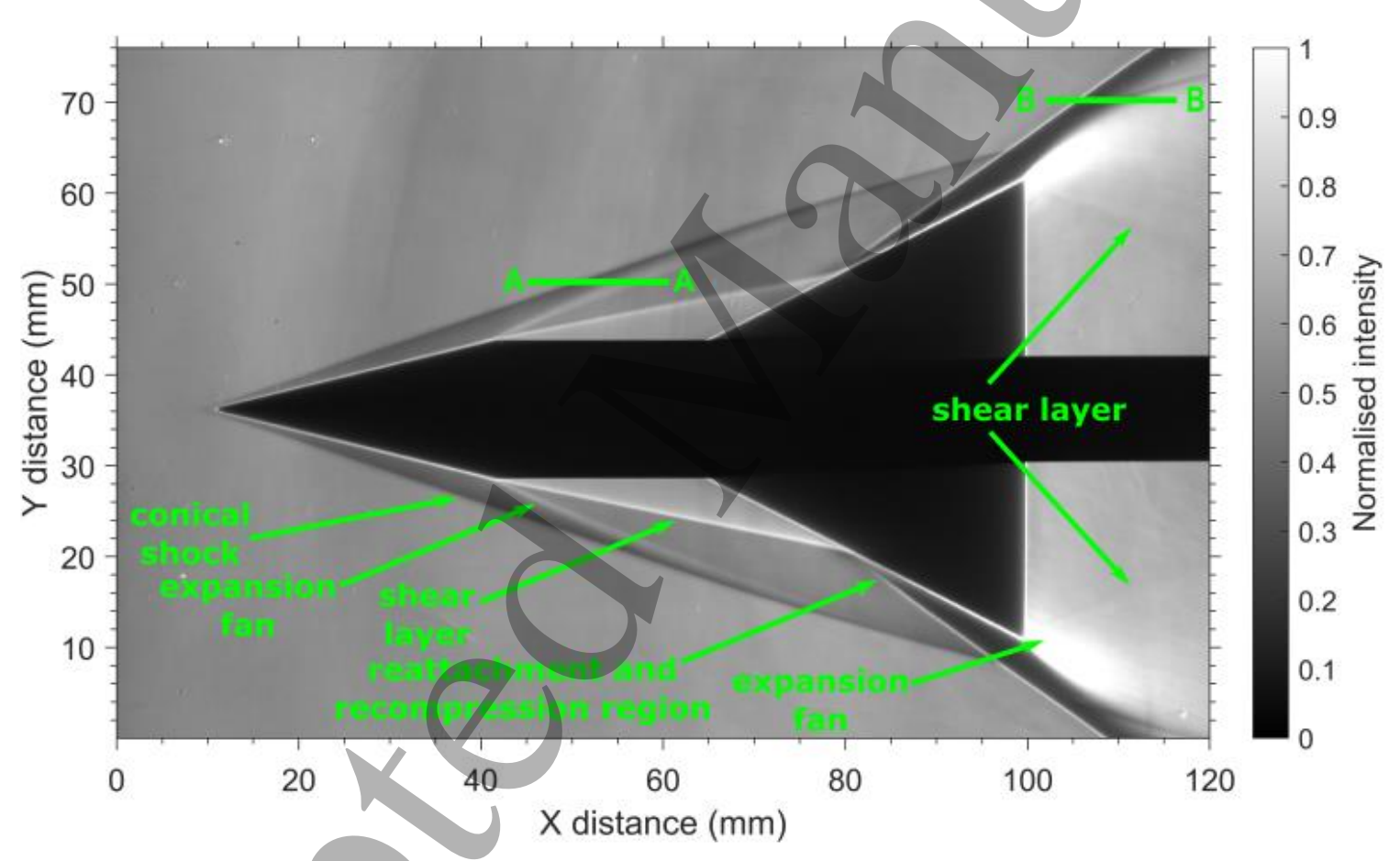

Figure 4: Flared-cone schlieren image, high- density case

The flared region of the model provides the greatest challenge when attempting to visualise the flow around this model, defined as B-B on Figure 4. A very strong compression region is immediately followed by a strong expansion region. The flow here is beyond the upper and lower limits of the dynamic range set by the physical knife edge in optical schlieren. Resolving these features is achievable but requires increased knife edge cut-off and multiple 
tunnel runs whilst sacrificing resolution of the weaker conical shock and shear layer on extract A-A. Increasing the cut-off of the knife edge ultimately results in an infinitely sensitive system, albeit over zero dynamic range.

\subsection{BOS optimisation}

The ideal algorithm to obtain the BOS images must preserve the strong discontinuity sharpness of the shock features and minimise the random freestream displacement noise. Reducing the freestream noise demands larger windows; typically smearing out the shock features of interest by adding processing blur. The optimal processing algorithm is an acceptable trade-off between shock capturing and minimal acceptable noise level. The highdensity flow condition was chosen to perform the processing study for the images as it would cause the largest background pattern pixel displacement.

Three performance metrics were examined; peak shock displacement, signal-to-noise ratio (SNR) and minimum 95\% shock rise distance. The SNR is defined as the ratio of the peak shock displacement and standard deviation of freestream noise. The $95 \%$ rise is defined as the minimum distance for the normalised shock displacement to rise from 5 to $95 \%$ of its final value.

The DIC processing in DaVis utilises circular windowing to correlate the displacements between the image pairs. Seven pixel windowing sizes were chosen, ranging from a very fine 5 pixel diameter up to an oversized 199 pixel diameter; a constant 5 pixel window overlap, equivalent to a PIV step, was chosen to maximise the output resolution, which remains independent of the windowing size [22]. Larger windowing sizes would have reduced the BOS spatial resolution further.

Figure 5 shows a line extract through point A-A (Figure 4) of the $\Delta \mathrm{Y}$ displacements through the conical shock. The processing window sizes of 5, 9, and 19 all capture the peak displacement value of approximately 2.3 pixels. Larger windows begin to reduce the peak displacement; displaying processing blur. 


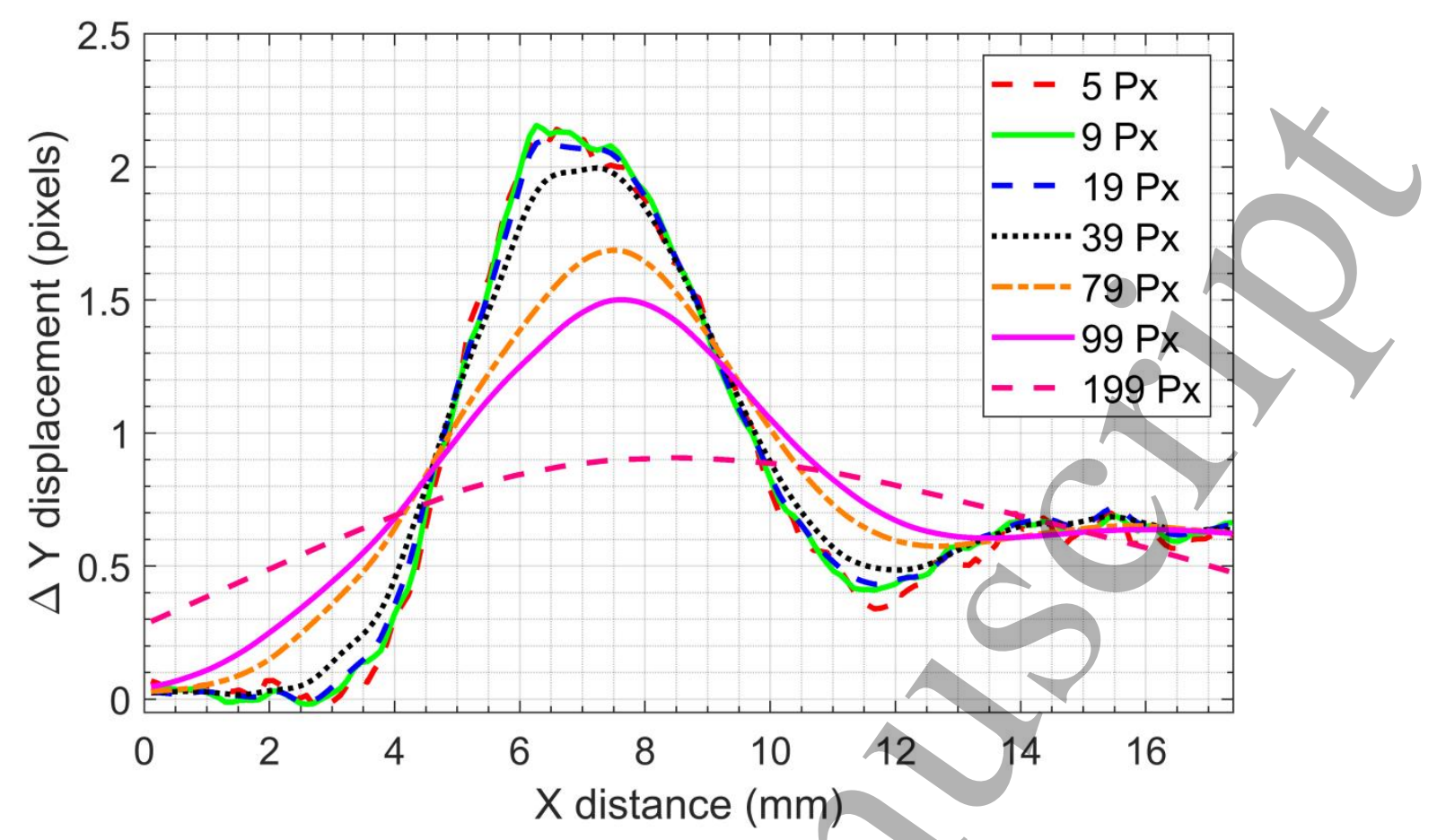

Figure 5: Effect of processing window size on vertical pixel displacement through the conical shock extract A-A

For each of the processing window cases the standard deviation of an area in the undisturbed freestream 100 pixels square was calculated. The vector displacement magnitude of random freestream displacement and peak shock displacements for the seven processing windows are compared in Figure 6, plotting random displacement on the lefthand $y$ axis, and magnitude of shock displacement on the right-hand axis. The windowing size has very little effect on the random noise with its magnitude decreasing from 0.05 to 0.02 pixels, slightly above the minimum detectable pixel displacement estimated by DaVis of 0.015 pixels. The shock displacement magnitude is significantly affected, decreasing almost six times from a maximum of 5.082 at the 9 pixel window to a value of 0.851 for the 199 pixel window.

The SNR of the first five processing windows is compared in Table 3; increasing from 93.3 at the 5 pixel window, reaching a peak of 161.2 at the 39 pixel window, and decreasing significantly to 39.2 at the largest 199 pixel windows. 
To quantify the effect of the window size on processing blur, the displacement profile normal to the $19.8^{\circ}$ oblique shock is taken through point A-A. The profile of normalised displacement magnitudes, $|\Delta N|$, through the shock for the 19 pixel window is plotted in Figure 7 . The 5 pixel window is highly sensitive to random noise pixel displacement, noted by the longer rise distance of $0.728 \mathrm{~mm}$, greater than the value for the 9 and 19 pixel windows. The 9 and 19 pixel window profiles are very closely matched, with a difference of $.047 \mathrm{~mm}$ between them. The shock capturing lag increases for the 39 and significantly for the 79 pixel window. Additionally for the 79 pixel window, the peak of the shock displacement was seen to move aft past the first four windows and clustered around the $2.7 \mathrm{~mm}$ to $2.9 \mathrm{~mm}$.

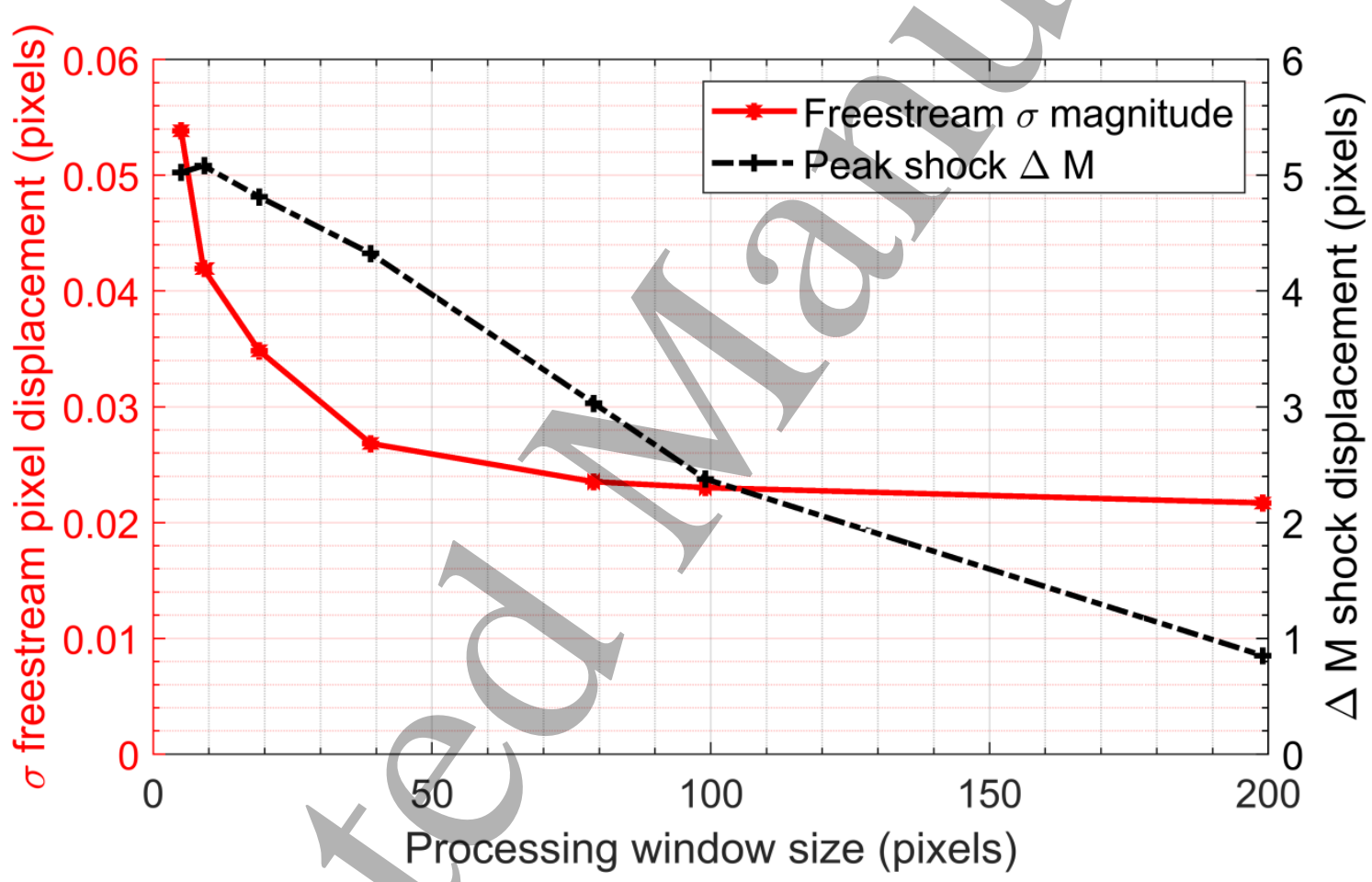

Figure 6: Effect of processing window size on magnitude of freestream random noise (left) and peak shock displacement magnitude (right) 


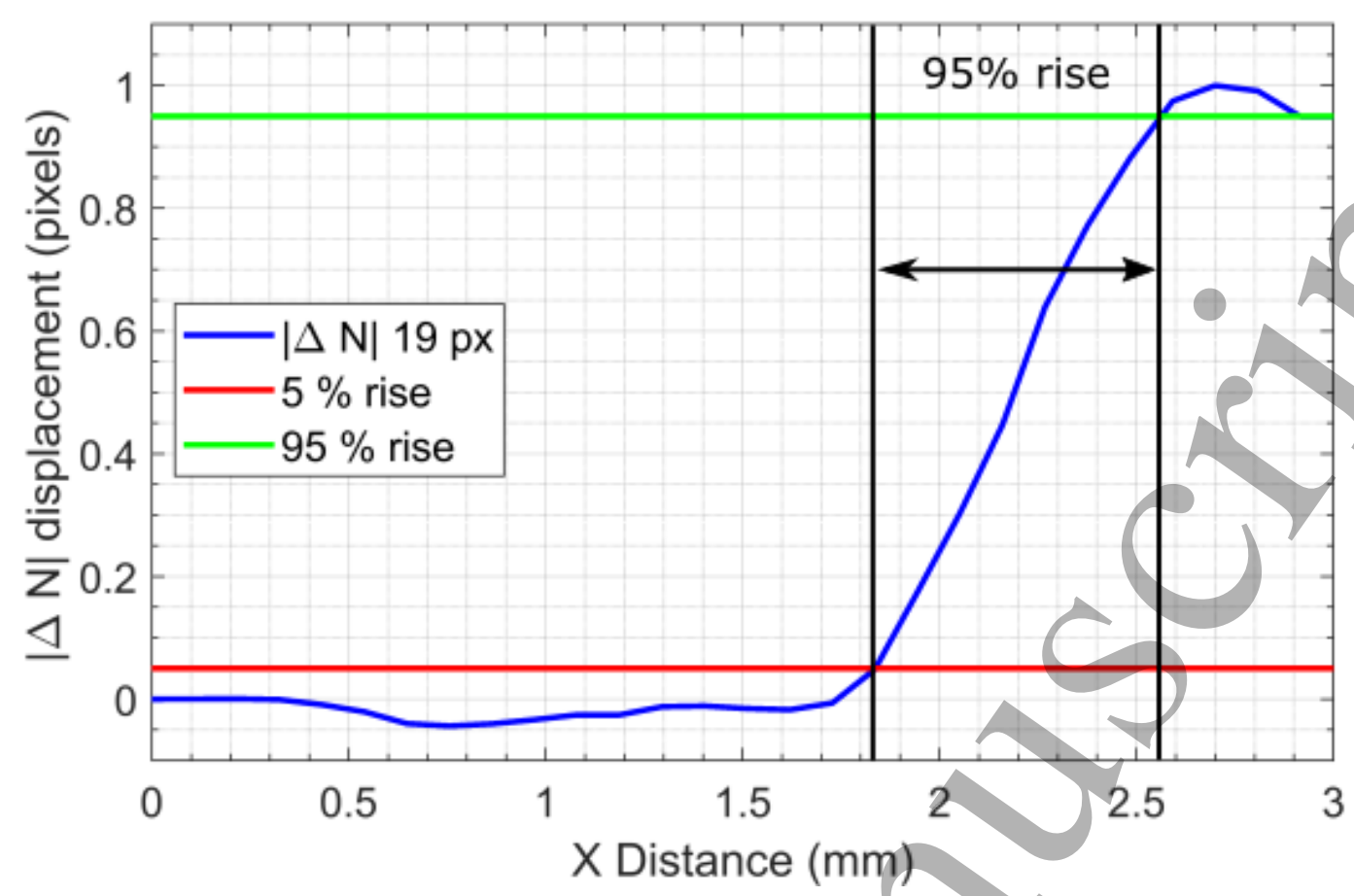

Figure 7: Profile of the pixel displacement normal to the conical shock processed with the 19 pixel window showing $95 \%$ rise distance $(\mathrm{mm})$

Table 3: Processing metric values

\begin{tabular}{|c|c|c|c|c|c|}
\hline Window Size & 5 & 9 & 19 & 39 & 79 \\
\hline Peak Displacement (pixels) & 5.020 & 5.082 & 4.814 & 4.325 & 3.031 \\
\hline SNR & 93.290 & 121.200 & 138.100 & 161.200 & 128.800 \\
\hline $95 \%$ shock rise (mm) & 0.728 & 0.679 & 0.726 & 0.837 & 1.259 \\
\hline
\end{tabular}

No single processing window size achieves all three performance metrics; however the 19 pixel window is the best compromise of the first three windows which closely agreed in the shock-capturing plot of Figure 5. Windowing sizes beyond 39 pixels all showed a decrease in performance metric values. The 5 pixel window was discounted as it is overly-sensitive to random displacements in the freestream. Although the 9 pixel window achieves the peak displacement, the SNR is lower than that of the 19 pixel window, with little difference in the $95 \%$ rise distance. The 19 pixel window was therefore selected as the optimum compromise and used to process the remainder of the BOS results. 


\subsection{BOS synthetic knife edge results}

In Figure 8 a) and b) the equivalent BOS and schlieren output for the high-density case are shown. Due to the processing window overlap the spatial resolution of the BOS images is five times lower than that of the schlieren images. However, despite this loss in spatial resolution the BOS $\Delta \mathrm{X}$ image captures all of the flow features described in the schlieren vertical knife edge image of Figure 4 and also in the comparable schlieren image below in Figure $8 b)$.

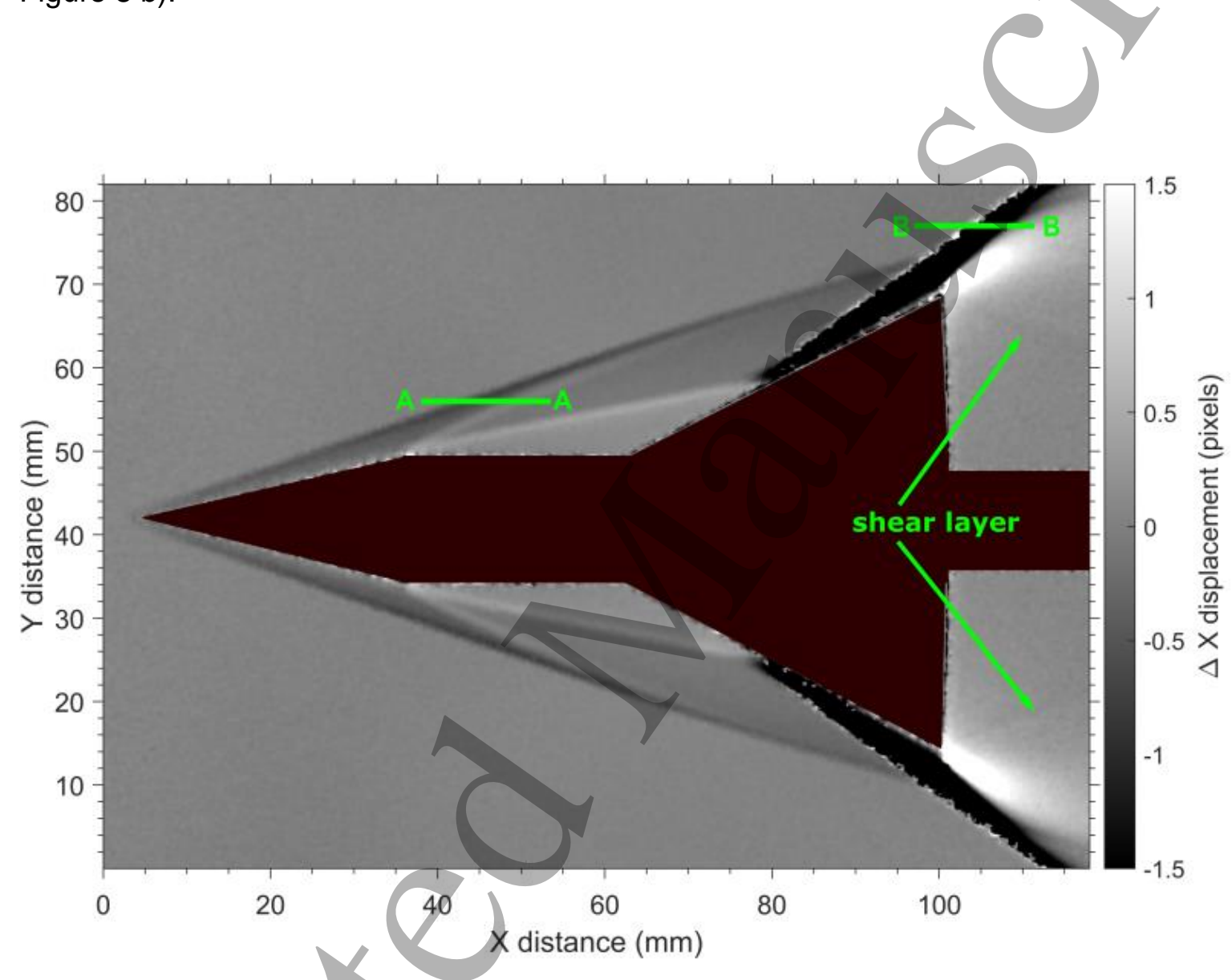

a) BOS horizontal displacements, $\Delta \mathbf{X}$ 


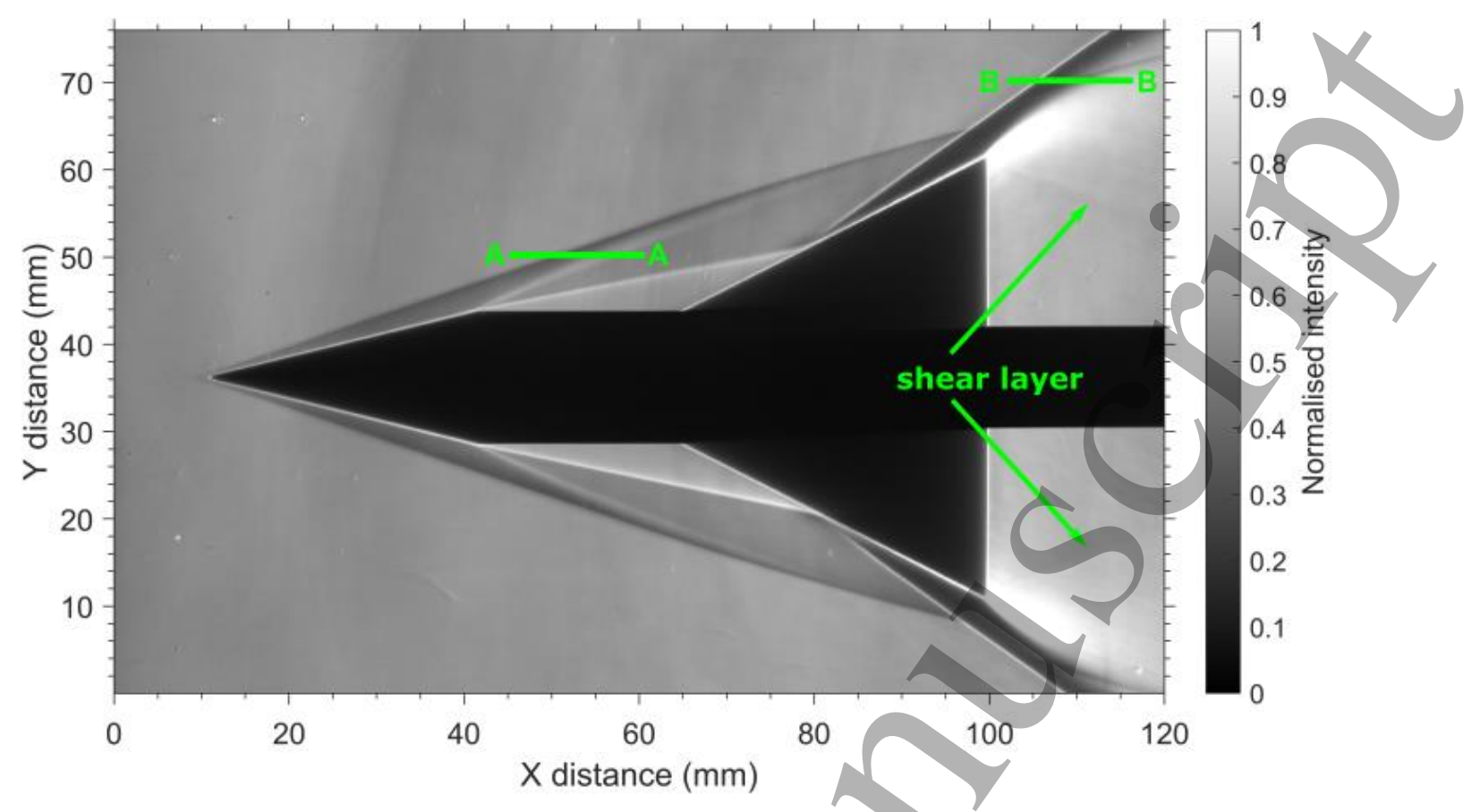

b) equivalent vertical knife edge schlieren results

Figure 8: High-density results: BOS horizontal displacement $\Delta \mathrm{X}$, equivalent vertical knife edge schlieren results

For the $\Delta \mathrm{X}$ results limiting displacement values of -1.5 and +1.5 pixel best captured the range of shock features surrounding the model. The displacements in $\Delta \mathrm{Y}$ are stronger than $\Delta \mathrm{X}$, affording a display limit range of \pm 3 for Figure $10 \mathrm{a}$ ), with an upper limit of 3.5 set for $\Delta \mathrm{M}$ in Figure $9 \mathrm{~b}$ ). This value is sufficient to discern the weaker shock features immediately following the expansion fan region at the tip of the rear flare.

The conical shock and the immediately following expansion fan are captured in the results of both techniques. The extract at B-B captures the strong recompression shock reflected from the rear flare of the model. The expansion fan region is smaller in Figure $9 \mathrm{~b}$ ), BOS $\Delta \mathrm{M}$ than the equivalent area of Figure $9 \mathrm{a}$ ) and Figure $8 \mathrm{a}$ ), BOS $\Delta \mathrm{X}, \Delta \mathrm{Y}$ due to the user set limits. The expansion fan in the schlieren image of Figure 8 b) is clipped at the upper limit of the camera sensor due to the fixed $50 \%$ knife edge cut-off, but the increased dynamic range of BOS for the current display ranges affords a greater insight to this very strong flow region. 
The area behind the rear flare of the model contains weak base-flow shear-layer features; these are annotated, and are weakly observable in the schlieren image of Figure $8 \mathrm{~b}$ ). The displacement $\Delta \mathrm{X}$ range set for the BOS in Figure 8 a) renders both shear layers, faintly observable. In Figure 9 a) and b) both pairs of shear layers are observable in the images;

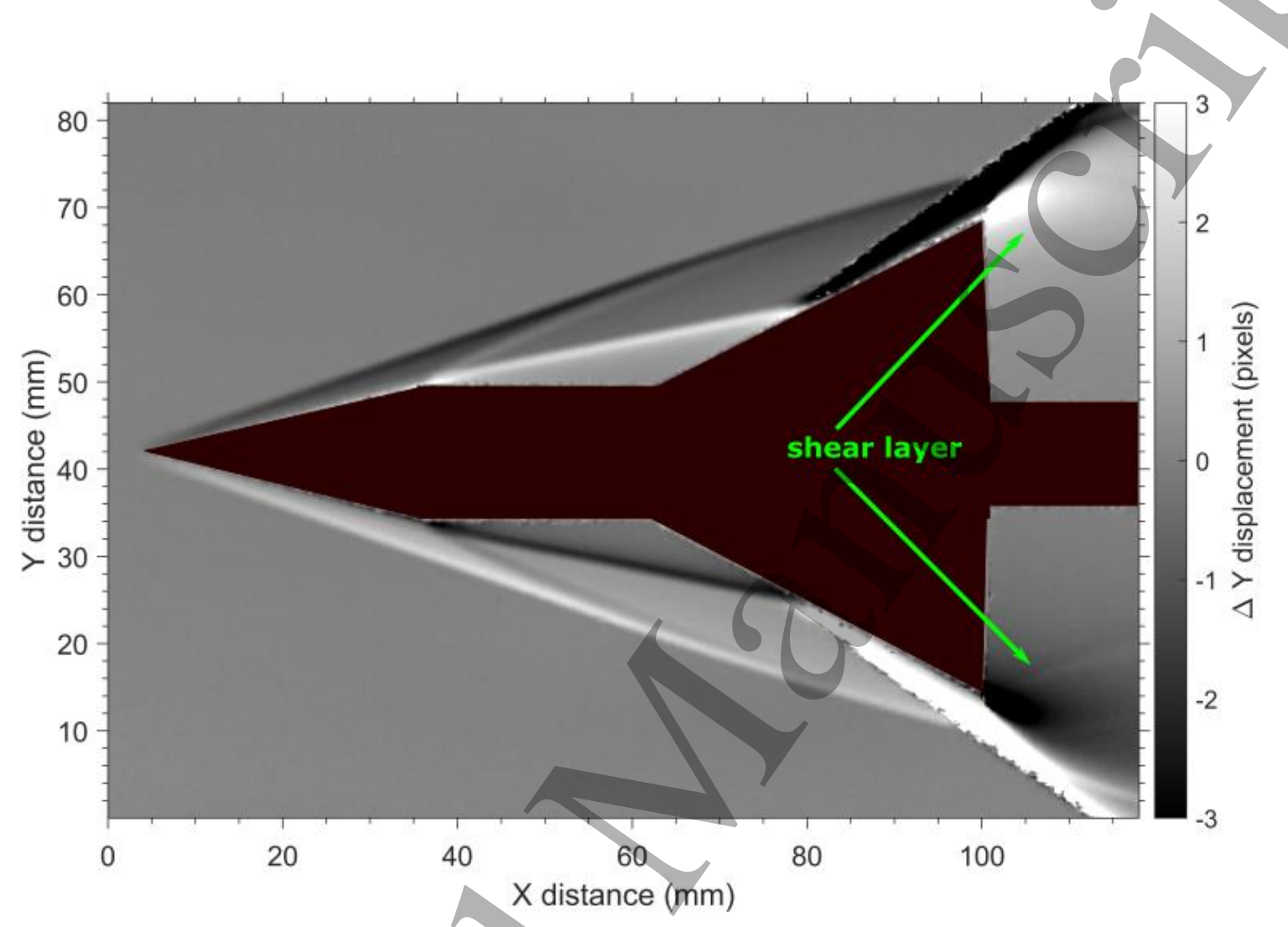

a) BOS vertical displacements, $\Delta Y$ 


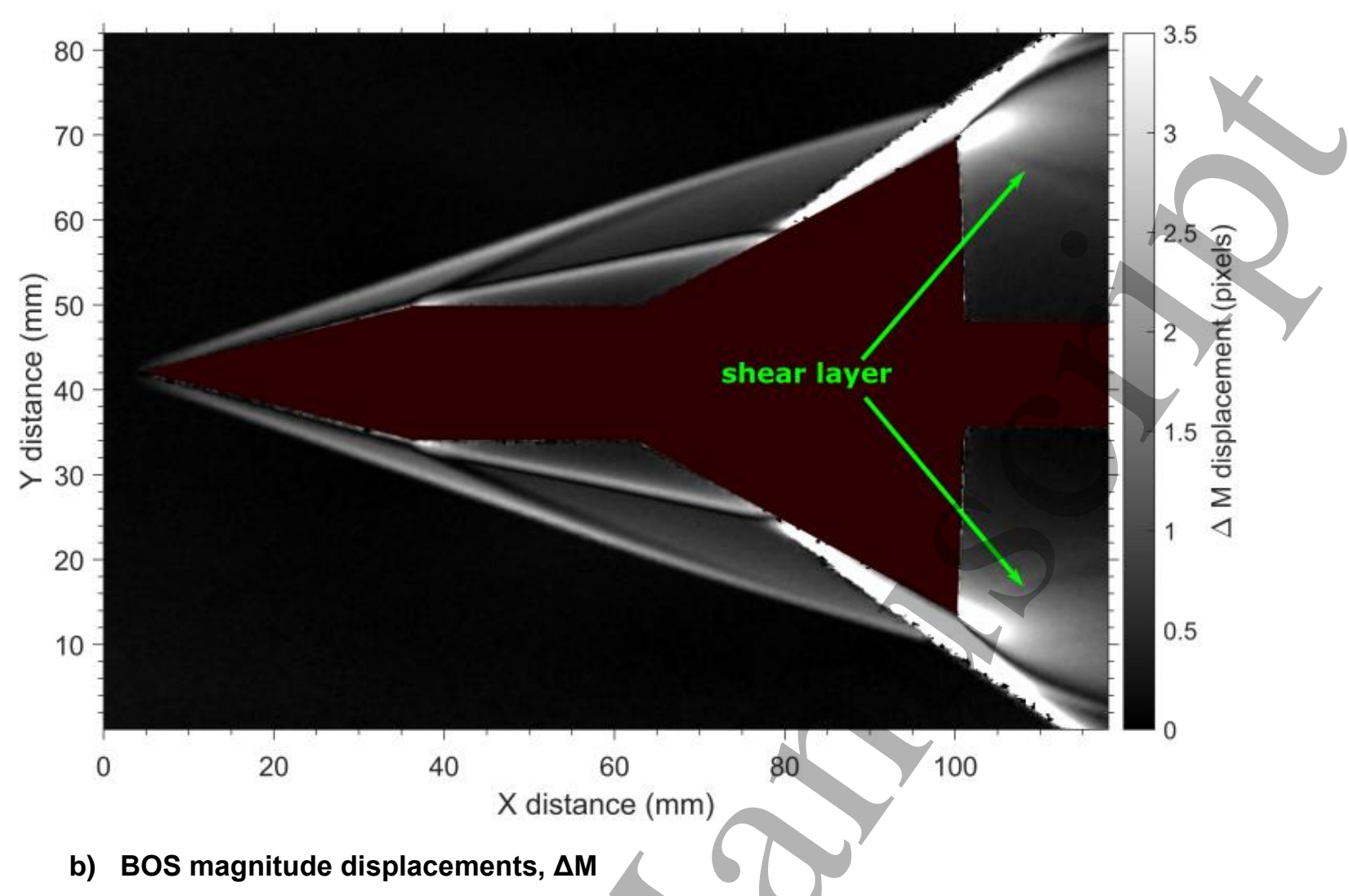

Figure 9: High density BOS vertical displacement, $\Delta \mathrm{Y}$ and displacement magnitude, $\Delta \mathrm{M}$

The ability to resolve BOS $\Delta \mathrm{X}$ and $\Delta \mathrm{Y}$ displacements to a custom synthetic knife edge orientation are described in Figure 10. Resolving the displacements arrays as normal vector, $\Delta \mathrm{N}$ allows the user to set any desired knife edge angle. An angle of zero obtains an equivalent output as displaying $\Delta Y$ with a positive colour map range. Setting the theta value to $-180^{\circ}$ inverts the colour map, producing $-\Delta \mathrm{Y}$. To demonstrate this, the $\Delta \mathrm{N}$ displacements were resolved to a custom angle of -129.8 degrees, shown in Figure 11. 


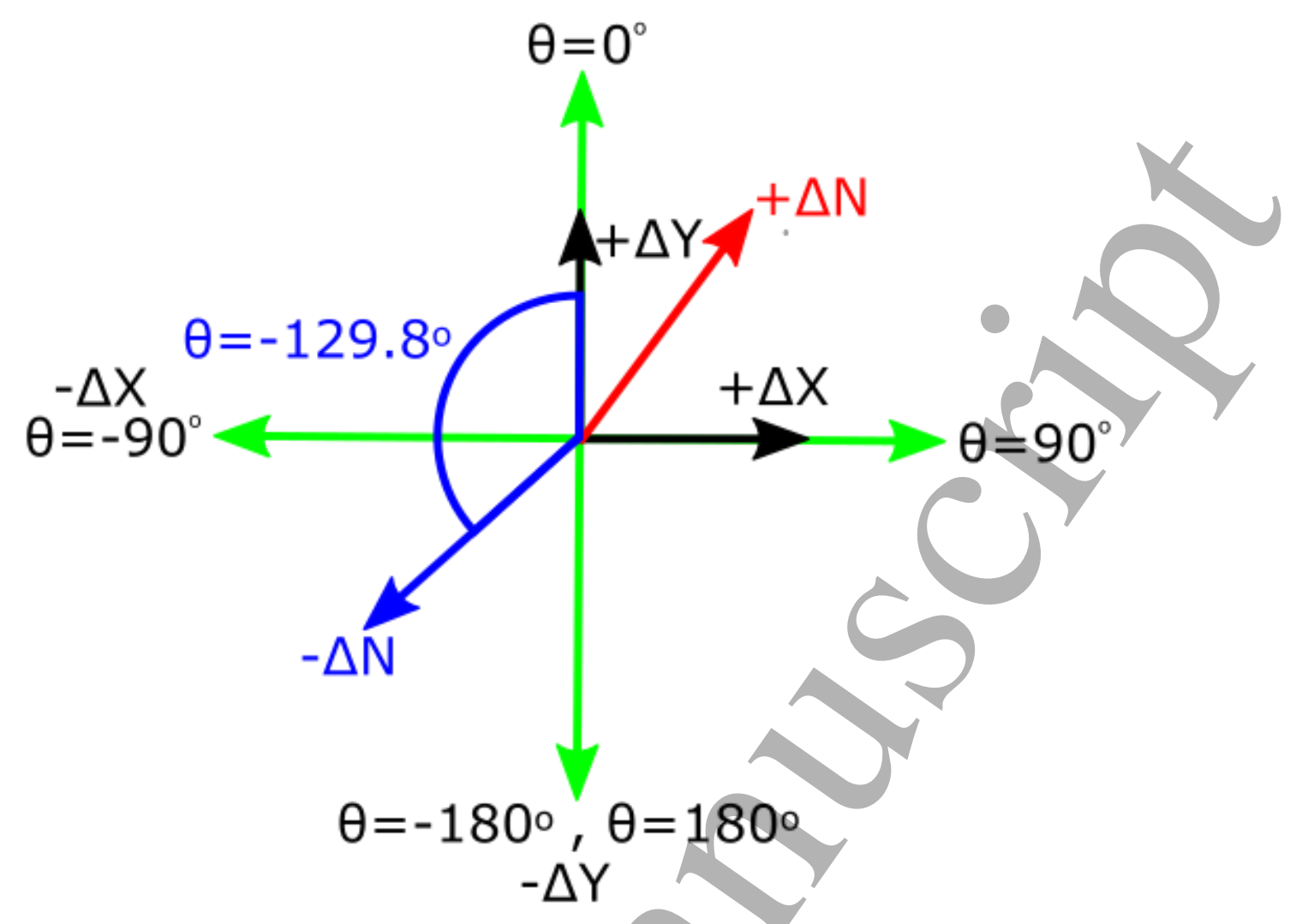

Figure 10: Synthetic knife edge coordinate system

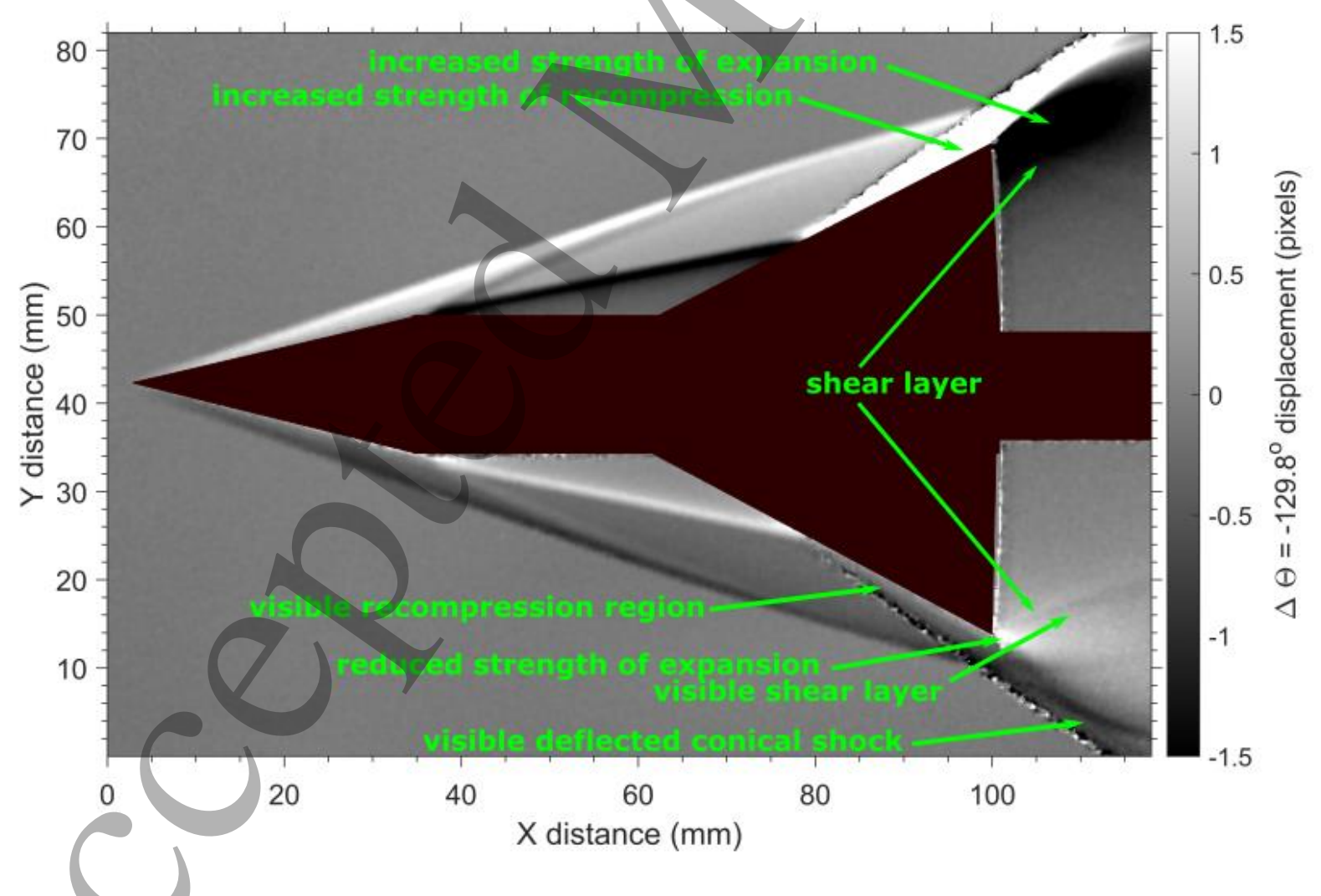

Figure 11: High-density BOS synthetic knife edge $-129.8^{\circ}$ angle resolved displacements 
The display range has been set the same as in Figure 8a) to show the weaker shear layer features that had been obscured on the base region behind the rear flare. The colour map range is inverted when compared to Figure 9 a). The custom orientation removes displacements aligned normal to the position of the knife edge. This reduces the intensity of the recompression region behind the lower shock, and also reduces the intensity of the expansion fan immediately following. The equivalent flow features on the upper region of Figure 11 have grown in strength for the fixed limits of the linear colour map. Of note is the obscuring to the shear layer on the upper half, but the increase strength of the shear layer on the lower surface.

The combination of synthetic knife edge orientation demonstrated in Figure 11 and cut-off range enables BOS to be used as a pathfinding diagnostic tool to inform the knife edge position and cut off of higher spatial resolution and reduced blur of optical schlieren. From a single BOS test arrangement the experimentalist is free to trial any combination of knife edge orientation which will reduce the number of "trial and error" tunnel runs to optimise the schlieren optics.

\subsection{Comparison of BOS and schlieren}

The results of the schlieren and BOS techniques obtained in section 3.2 and 3.3 are compared to determine the sensitivity to a variety of flow features generated by the model.

High-speed video taken at $10 \mathrm{kHz}$ of the conical shock position was used to characterise motion blur. The standard deviation of the shock position as measured by the maximum intensity, per row, on an x-t diagram was sub-pixel, approximately 0.4487 pixels.

The schlieren and BOS image stacks were aligned in Matlab to allow for feature comparison. The flow field around the flared-cone is not perfectly steady; the position at which the shear layer impinges onto the rear flare of the cone moves during a tunnel test. This manifests as a "wandering" of the recompression shock angle. This occurs for all three density cases and during both techniques; the image registration is able to align the defined points of the model 
and perform translation, scaling and rotation of the image stack. The unsteady shear-layer reattachment on the rear flare results in movement of the shock positions during a test run. The oscillation of the reattachment coupled with finite images acquired during the test prevents precise matching of shock positions. The movement of the recompression shock and expansion fan is typically $0.6 \mathrm{~mm}$.

In Figure 12 shock profile extracts through A-A and B-B identified Figure 8 a) for BOS and in Figure $8 \mathrm{~b}$ ) for schlieren was compared for the three values of freestream density. These are non-dimensionalised into arbitrary units; allowing the shock-profile from both techniques to be compared on the same spatial and displacement scale. Arbitrary schlieren intensity shift on the left-hand $y$ axis and arbitrary BOS displacements on the right-hand y axis. BOS $\Delta \mathrm{X}$ had the freestream random noise removed and the values inverted to make all displacements positive. The profiles were then non-dimensionalised with respect to the peak displacement for the high-density case. For the schlieren extracts the freestream intensity value was removed and profile divided by the peak intensity value for the high density case; producing negative intensity values after the conical, and recompression shocks.

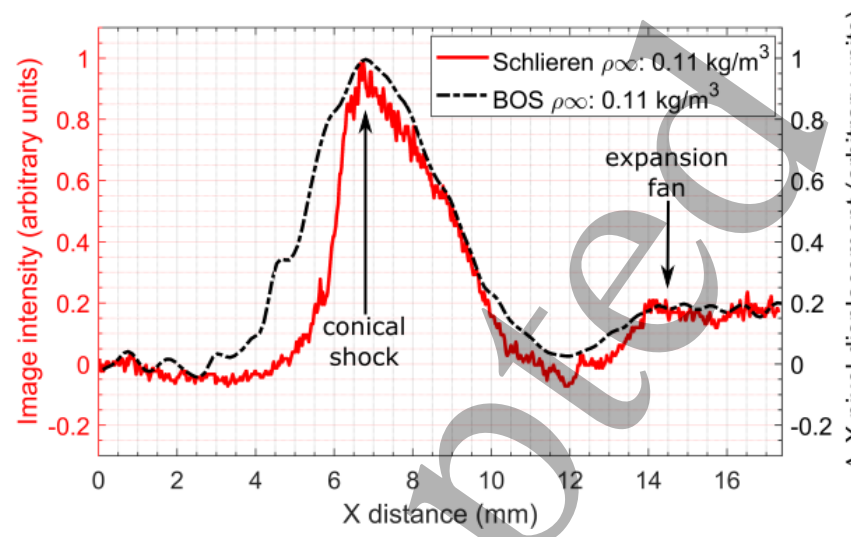

a) high-density extract A-A

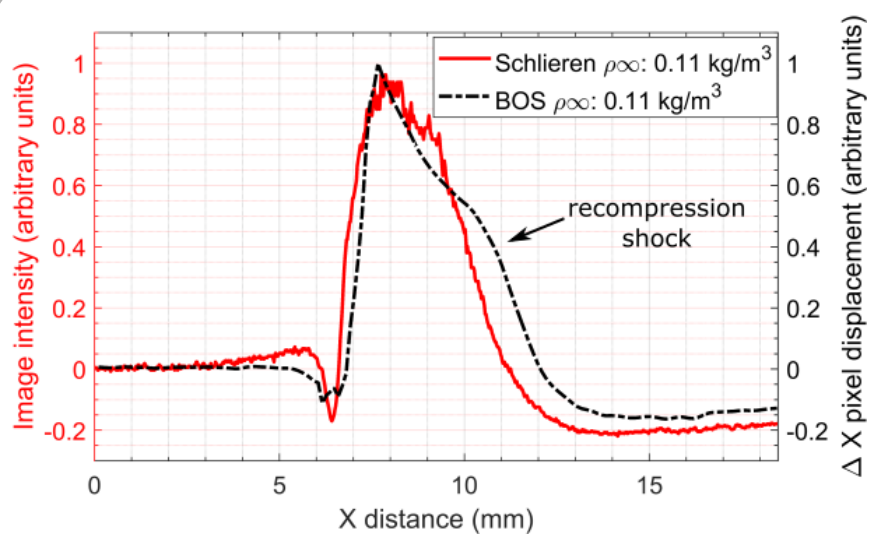

d) high-density extract B-B 


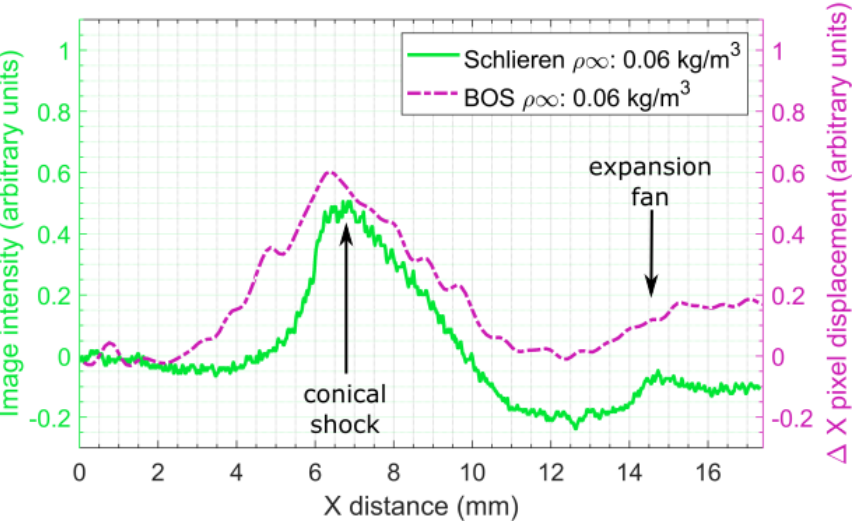

b) medium-density extract $A-A$

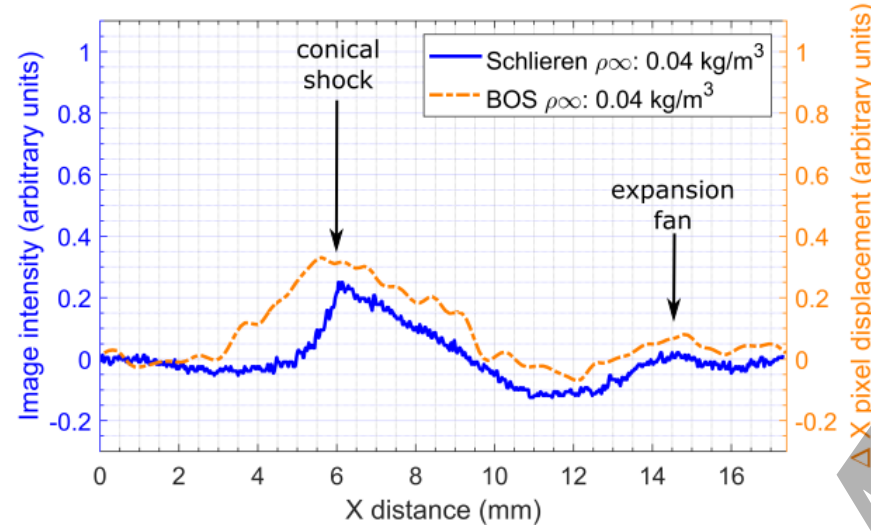

c) low-density extract A-A

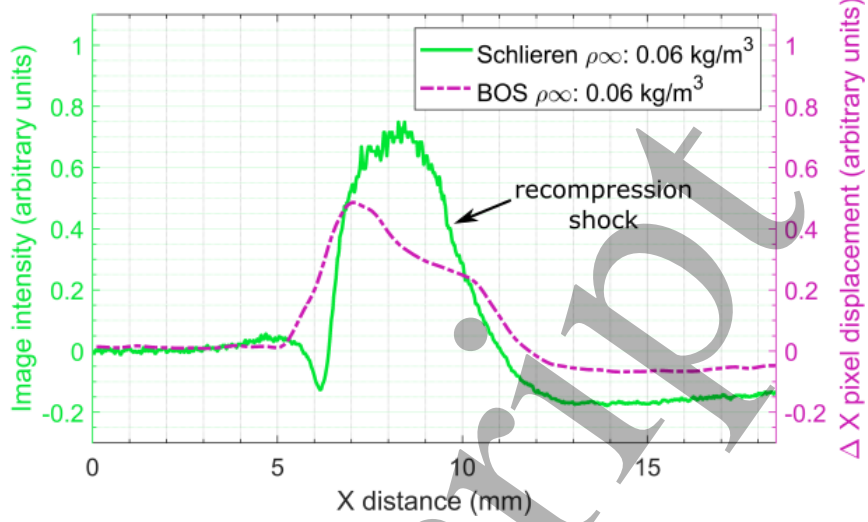

e) medium-density extract B-B

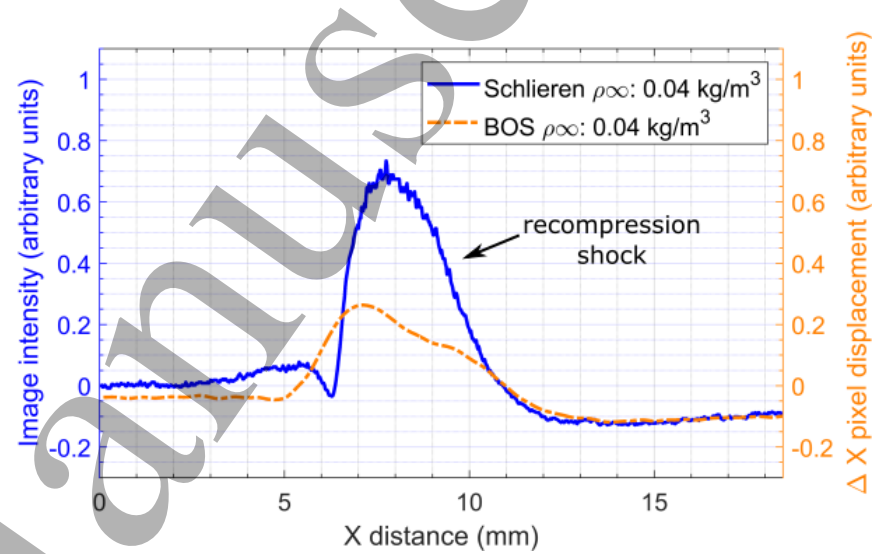

f) low-density extract B-B

Figure 12: Arbitrary schlieren intensity and BOS $\Delta \mathrm{X}$ displacement extracts through shock extract A-A, and shock extract B-B.

The shock profiles A-A for schlieren and BOS (Figure 12 a-c) exhibit a maximum peak corresponding to the strong discontinuity through the shock. A second peak is produced by the expansion fan where the leading cone meets the shoulder of the model. The BOS profile extracts all demonstrate a leading displacement rise ahead of the conical shock, which is not present for the corresponding schlieren results. Evaluation of PIV tracer particles through an oblique shock by Glazyrin et al. [23] and Ragni et al. [24] discussed reasons for velocity jump blur ahead of the shockwave front; other deformation effects included optical distortion of the PIV image as a result of refraction of light due to the variation of density. Shocks with sufficient curvature, which applies to the entirely curved model flow field, produced additional displacement of the PIV tracer particles. The schlieren results of the shock rise have a higher spatial resolution than BOS as they do not feature the displacement of the 
background smeared ahead of the true position of the shock, reproducing the effect noted in PIV. Furthermore, the longer exposure time of the BOS images as opposed to the schlieren will incur some motion blur.

The schlieren results in the right hand column of Figure 12 exhibit a kurtosis of zero around the region of the recompression shock. The medium and low-density cases/Figure 12 e) and f) the schlieren peak displacement through the recompression shock reduces slightly, whereas the BOS results decrease markedly compared to the schlieren. The physical knifeedge imposed fixed-sensitivity limits the dynamic range and thereby clips recompression regions. The BOS extracts through the recompression shock still exhibit the leading displacement rise seen in the A-A extracts, reducing their overall spatial resolution. The synthetic knife edge cut-off affords more information about the flow in this region than the schlieren. The BOS results in Figure $12 \mathrm{~d}$-f) show a maximum peak in $\Delta \mathrm{X}$ displacement corresponding to the leading edge of the shock, but this gradually decreases in strength towards the rear of the shock region in the $+X$ axis.

The BOS configuration has an increased dynamic range compared to the schlieren results which are clipped due to the fixed knife edge/and strength of the recompression shocks; however BOS has an overall lower spatial resolution exhibiting forward shock smearing due to the imposed longer exposure time than for schlieren. Comparing the global flow fields the BOS technique has at least equivalent sensitivity to the major flow features present around the model in Figure 4.

The BOS technique sensitivity to freestream density was compared through the extract A-A. It is evident from Snell's law that a reduction in density promotes a corresponding reduction in background shift, noted in Figure 12 a-c). The vector magnitude $\Delta \mathrm{M}$ normalised to the high-density condition through A-A is plotted on Figure 13 against freestream density on the $\mathrm{X}$ axis. The trend is well fitted by a first order polynomial, confirming a linear relationship. 


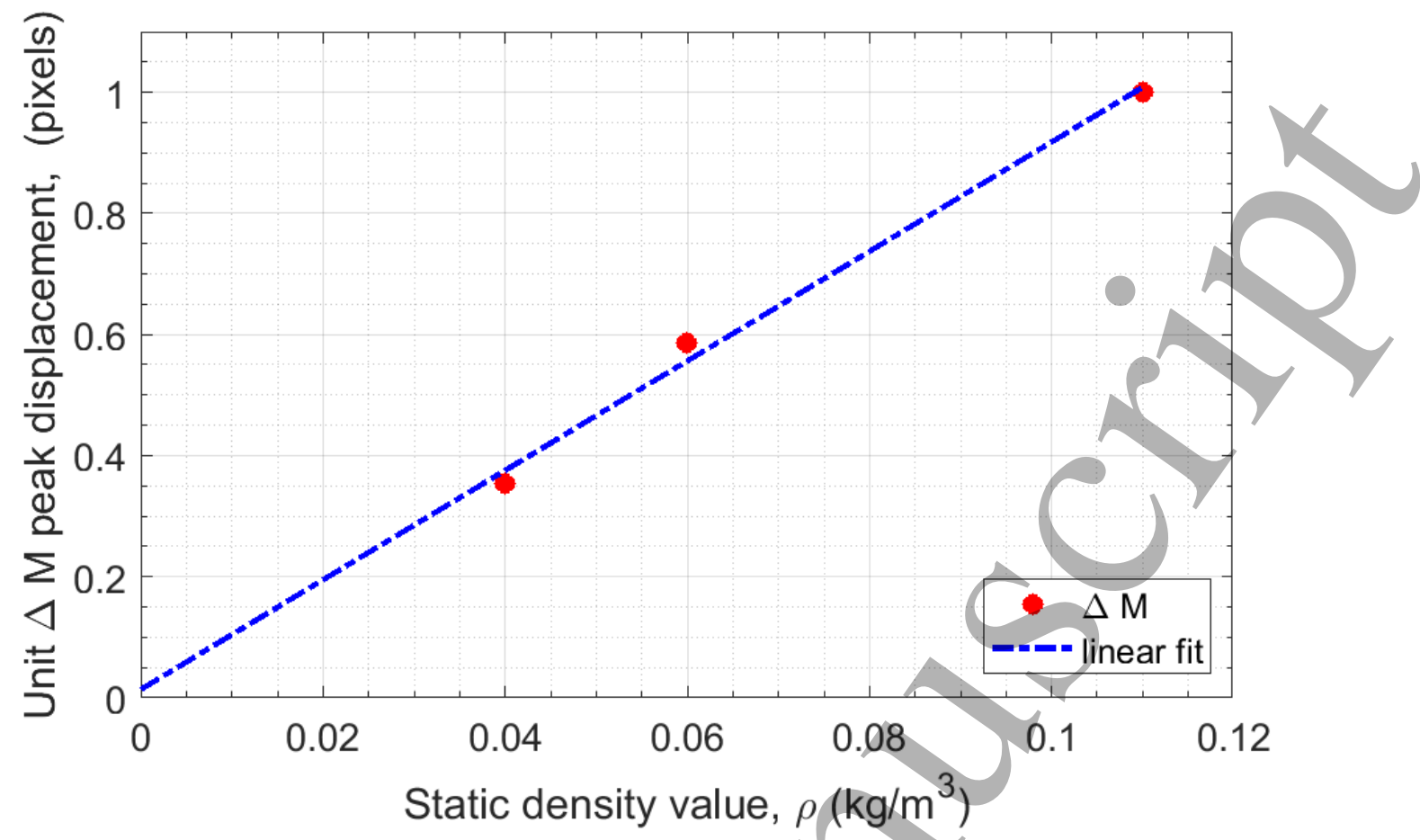

Figure 13: relationship between extract $A-A$ shock displacement magnitude $\Delta M$ and freestream density

\section{Conclusions}

BOS and schlieren data has been acquired for a complex flow field. The BOS results have a lower spatial resolution than the schlieren images due to the processing, exposure time and leading displacement shock rise. Despite this, the technique resolved the major flow features obtained from the comparison schlieren images.

Three processing optimization metrics were proposed; peak shock displacement, SNR and minimum 95\% shock rise distance. From the processing study for a fixed window overlap and seven windowing sizes the best was the 19 pixel window achieving the highest SNR of the first three windowing sizes and negligible difference in the $95 \%$ rise distance to the 9 pixel window. BOS results for three comparable traditional schlieren knife edge orientations were obtained, with the synthetic knife edge enabling a higher dynamic range than that of the schlieren, for all the $\Delta \mathrm{X}, \Delta \mathrm{Y}$ and $\Delta \mathrm{M}$ BOS results; due to the fixed sensitivity limits imposed by the compromise $50 \%$ schlieren cut-off. 
The BOS results were resolved to a custom $-129.8^{\circ}$ orientation to demonstrate the ability of the technique to set custom synthetic knife edge orientations. The synthetic knife edge can inform the positioning and cut-off of a physical knife edge for optical schlieren data to benefit from high-spatial resolution images. This is not possible in the conventional schlieren technique without changing the physical knife edge setting and re-running the tunnel.

Comparison of non-dimensionalised shock profiles for BOS and schlieren at two locations were compared for the three values of freestream static density. The lower spatial resolution of the BOS was evidenced by a leading displacement rise ahead of the conical shock due to optical distortion of the background and motion blur.

The normalised BOS vector magnitude $\Delta \mathrm{M}$ was examined through the conical shock against freestream density, with a well-fitted linear trend between increasing density and increasing displacement.

This study demonstrates the complimentary nature of the BOS technique, alongside traditional optical schlieren as powerful tools for the study of compressible flows. Utilisation of BOS to inform schlieren knife edge orientations and cut off amount through the use of a synthetic knife edge from a single test image reduces costly tunnel time to fine-tune a physical optical set up.

Examination of multiple flow features occurring in quasi-unsteady flow conditions can be investigated post-test in BOS on a single image. This is not achievable in schlieren which would require repeat tests and knife edge orientations with dissimilar flow conditions to those desired. 


\section{Acknowledgements}

This work was funded by the European Space Agency under a Network Partnering Initiative scheme. Grant Number 456. The authors would like to acknowledge the European Space Agency for their support and funding for this project.

\section{References}

[1] G. E. A. Meier, "Hintergrund-Schlierenmeßverfahren für räumliche Dichtefelder," Deutsche Patentanmeldung DE 19942856 A1.

[2] M. Raffel, H. Richard, and G. E. A. Meier, "On the applicability of background oriented optical tomography for large scale aerodynamic investigations," Exp. Fluids, vol. 28, no. 5 , pp. $477-481,2000$.

[3] B. R. Sutherland, S. B. Dalziel, G. O. Hughes, and P.F. Linden, "Visualization and measurement of internal waves by synthetic schlieren. Part 1. Vertically oscillating cylinder," J. Fluid Mech., vol. 390, pp. 93-126, 1999.

[4] S. B. Dalziel, G. O. Hughes, and B. R. Sutherland, "Whole-field density measurements by synthetic schlieren," Exp. Fluids, vol. 28, no. 4, pp. 322-335, 2000.

[5] F. Sourgen, F. Leopold, and D. Klatt, "Reconstruction of the density field using the Colored Background Oriented Schlieren Technique (CBOS)," Opt. Lasers Eng., vol. 50, no. 1, pp. 29-38, 2012.

[6] M. Ota, K. Hamada, H. Kato, and K. Maeno, "Computed-tomographic density measurement of supersonic flow field by colored-grid background oriented schlieren (CGBOS) technique," Meas. Sci. Technol., vol. 22, no. 10, p. 104011, 2011.

[7] F. A. Mier and M. J. Hargather, "Color gradient background-oriented schlieren imaging," Exp. Fluids, vol. 57, no. 6, pp. 1-14, 2016.

[8] H. Richard and M. Raffel, "Principle and applications of the background oriented schlieren (BOS) method," Meas. Sci. Technol., vol. 12, no. 9, pp. 1576-1585, 2001.

[9] J. Raffel, M., Willert, C.E., Wereley, S. T., Kompenhaus, Particle Image Velocimetry, 2nd ed. Berlin, Heidelberg: Springer Berlin Heidelberg, 2007.

[10] F. Leopold, "The Application of the Colored Background Oriented Schlieren technique (CBOS) to free-flight and in-flight measurements," ICIASF Rec. Int. Congr. Instrum. Aerosp. Simul, Facil., 2007.

[11] B. Atcheson, W. Heidrich, and I. Ihrke, "An evaluation of optical flow algorithms for background oriented schlieren imaging," Exp. Fluids, vol. 46, no. 3, pp. 467-476, 2009.

[12] M. Raffel, "Background-oriented schlieren (BOS) techniques," Exp. Fluids, vol. 56, no. 3, pp. 1-17, 2015.

[13] T. Fisher, M. K. Quinn, and K. Smith, "Free-Flight Testing of Hypersonic Edney Shock Interactions," 2018 Aerodyn. Meas. Technol. Gr. Test. Conf., 2018. 
[14] P. Bean, J. T. Heineck, D. Banks, E. T. Schairer, and E. A. Haering, "Background Oriented Schlieren (BOS) of a Supersonic Aircraft in Flight," no. June, 2016.

[15] P. Manovski, J. Wehrmeyer, K. Scott, B. Loxton, H. Quick, S. Lam, M. Giacobello, “A Performance Comparison between Classical Schlieren and Background-Oriented Schlieren," 18th Int. Symp. Appl. Laser Imaging Tech. to Fluid Mech., 2016.

[16] T. Kirmse, J. Agocs, A. Schröder, M. J. Schramm, S. Karl, and K. Hannemann, "Application of particle image velocimetry and the background-oriented schlieren technique in the high-enthalpy shock tunnel Göttingen," Shock Waves, vol. 21, no. 3, pp. 233-241, 2011.

[17] D. Ramanah, S. Raghunath, D. J. Mee, T. Rösgen, and P. A. Jacobs, "Background oriented schlieren for flow visualisation in hypersonic impulse facilities," Shock Waves, vol. 17, no. 1-2, pp. 65-70, 2007.

[18] W. Sutherland, "LII. The viscosity of gases and molecular force," Philos. Mag. Ser. 5, vol. 36, no. 223, pp. 507-531, 1893.

[19] A. B. Gojani, B. Kamishi, and S. Obayashi, "Measurement sensitivity and resolution for background oriented schlieren during image recording," J. Vis., vol. 16, no. 3, pp. 201-207, 2013.

[20] Ames Research Staff, "Equations, Tables and Charts for Compressible Flow," Moffet Field, 1947.

[21] J. J. Bertin, Hypersonic Aerothermodynamics. New York: American Institute of Aeronautics \& Astronautics, 1994.

[22] M. Havermann, J. Haertig, C. Rey, and A. George, "PIV measurements in shock tunnels and shock tubes," Top. Appl. Phys., vol. 112, pp. 429-443, 2008.

[23] F. N. Glazyrin, I. V Mursenkova, and I. A. Znamenskaya, "PIV tracer behavior on propagating shock fronts," Meas. Sci. Technol., vol. 015302, p. 15302.

[24] D. Ragni, F. Schrijer, B. W. Van Oudheusden, and F. Scarano, "Particle tracer response across shocks measured by PIV," Exp. Fluids, vol. 50, no. 1, pp. 53-64, 2011. 